Erschienen in: Regula Julia Leemann, Christian Imdorf, Justin J.W. Powell, Michael Sertl (Hrsg.) (2016). Die Organisation von Bildung. Soziologische Analysen zu Schule, Berufsbildung, Hochschule und Weiterbildung. Weinheim und Basel, S. 166-184.

\title{
Organisatorisch gerahmte Interaktion. Überlegungen zu einer transsituativen Ethnographie der schulischen Bildung
}

\section{Tobias Röhl}

Was sich tagtäglich in den Klassenzimmern der Schulen abspielt, folgt bestimmten erkennbaren Mustern. Wenn man sich ethnographisch anschaut, was Lehrer und Schüler da miteinander veranstalten, wird schnell klar, dass man es mit einer spezifischen Form der Interaktion zu tun hat, die man aus eigener Erfahrung als Unterricht kennt. Nun kann man diese Interaktion aus sich selbst heraus erklären, indem man beobachtet, wie Lehrer und Schüler wechselseitig die Interaktion strukturieren und situativ Ordnung erzeugen. Damit blendet man aber oftmals die organisatorischen Vorbedingungen dieser Interaktion aus. Ich schlage im Folgenden vor, dem dadurch zu begegnen, dass man den Schulunterricht verlässt und nachzeichnet, wie verschiedene materielle Artefakte Unterricht vorstrukturieren und mit anderen organisatorischen Akteuren verbinden. Darin - so die These - besteht die organisatorische Rahmung der lokalen Unterrichtsinteraktion. Meine Überlegungen stützen sich auf eine eigene ethnographische Forschung zum mathematisch-naturwissenschaftlichen Schulunterricht in der gymnasialen Sekundarstufe; besonderes Augenmerk lag dabei auf der Rolle materieller Artefakte für die schulische Wissensvermittlung (Röhl 2013).

Zunächst arbeite ich heraus, was die ethnographische Perspektive für die Organisationsund Bildungsforschung leisten kann und an welche Grenzen sie dabei stößt (1.). Daran anschließend zeigt der Aufsatz, wie man der materiell vermittelten organisatorischen Rahmung der Unterrichtsinteraktion ethnographisch folgen kann (2.). Besondere Beachtung finden dabei architektonische Arrangements (Unterrichtsräume und schulisches Mobiliar) sowie kommerziell hergestellte Lehrmittel (etwa Demonstrationsexperimente) und ihre schrittweise schulische Aneignung und Zurichtung. Abschließend werden Konsequenzen der hier vorgeschlagenen ethnographischen Perspektive für die Erforschung schulischer Organisation(en) skizziert (3.).

\section{Ethnographie schulischer und außerschulischer Organisationen}

Die Organisationsforschung kennt die ethnographische Perspektive seit geraumer Zeit (siehe Whyte 1948), und sie gilt dort mittlerweile als etablierte Methode mit einer eigenen Zeitschrift (Journal of Organizational Ethnography, Emerald) sowie Hand- und Lehrbüchern (Ybema u. a. 2009; Neyland 2007). Die Organisationsethnographie folgt der Idee einer „eth- 
nography at home": Statt der Erforschung fremder Kulturen, geht es um die Erforschung spezialisierter Subkulturen innerhalb der eigenen Gesellschaft (Rosen 1991). Ethnographische Studien konnten beispielsweise zeigen, dass Organisationen nicht bloß rational gesteuerte Entitäten mit formellen Vorgaben sind, sondern die tägliche Arbeit in ihnen zu einem großen Teil auf informelle Regelungen zurückgreift (etwa Crozier 1964). In den Blick gerät dann das nur schwer formalisierbare implizite Wissen der Organisationsmitglieder. Ethnographische Forschung impliziert ferner eine „bottom up“-Perspektive auf Organisationen: Statt von organisationstheoretischen oder funktionalistischen Überlegungen auszugehen, zeichnet die Forschung nach, wie die Organisationsmitglieder Ordnung schaffen und den Abläufen um sie herum Bedeutung verleihen. Damit einher geht ein Misstrauen gegenüber der Idee der Steuerbarkeit der Arbeitsabläufe auf Ebene des Managements oder anderer Führungsorgane. Trotz dieser Skepsis gibt es Bemühungen, die Zusammenhänge zwischen verschiedenen Ebenen der Organisation nicht zugunsten einer interaktionistischen Fokussierung auf die lokale Situation aus den Augen zu verlieren. Unter Rückgriff auf Anselm Strauss untersuchen beispielsweise Nadai und Maeder (2008), wie die Leistung eines Unternehmens das Ergebnis von "Aushandlungen“ („,negotiations“; Strauss 1978) zwischen lokalen Akteuren, Organisationsstrukturen und gesellschaftlichen Diskursen ist. Organisationsethnographie zielt deshalb nicht bloß auf eine Ethnographie in, sondern auf eine Ethnographie der Organisation (vgl. Eberle/Maeder 2011), also auf klassische Fragen der Organisationsforschung: Wie werden Mitglieder rekrutiert? Wie werden Ziele der Organisation verfolgt und umgesetzt? Wie wird die Arbeit innerhalb der Organisation koordiniert und kontrolliert? Etc.

Auch innerhalb der Bildungsforschung finden sich zahlreiche ethnographische Studien: etwa zur Unterrichtsinteraktion mit all ihren impliziten Regeln und Abläufen (etwa Breidenstein 2006; Mehan 1979). Daneben ist u.a. die „peer culture“ der Schüler auf den Pausenhöfen, aber auch im Unterricht Gegenstand der Forschung (Kelle 1999; Corsaro 1985). Seltener berichten die Ethnographinnen und Ethnographen aus den Lehrerzimmern (Pille 2013), von Notenkonferenzen (Maier 2014 und in diesem Band), mündlichen Prüfungen oder Gesprächen zur Notenfindung (Kalthoff 1996). Die Stärke dieser Studien besteht in ihrer detailgenauen, analytischen Beschreibung dessen, was sich in Schulen abspielt. Der Fokus der Forschung liegt zumeist auf einzelnen Bereichen, Orten und Situationen, die aber nicht systematisch in Verbindung miteinander gebracht werden. Ungeklärt bleibt folglich, wie die einzelnen ethnographisch beschriebenen Situationen aneinander anschließen bzw. anschlussfähig gemacht werden.

Wichtig ist in diesem Zusammenhang, dass Schulunterricht kein voraussetzungsloses Zusammentreffen von Individuen, sondern "organizationally framed interaction" (Vanderstraeten 2001, S. 270ff.) ist. Die Schule und andere staatliche Organisationen (etwa die Kultusministerien) sorgen beispielsweise für die Einteilung der Schüler in Jahrgangsklas- 
sen, die Bereitstellung von Räumen und Lehrkräften, die Festsetzung von Lehr- und Stundenplänen, die asymmetrische Zuweisung von Rollen (Lehrer/in, Schüler/in) usw. Daneben sind auch eine Reihe von privatwirtschaftlichen Organisationen an dieser Rahmung des Unterrichts beteiligt: die Lehrmittelindustrie stellt Experimentierkästen und Anschauungsobjekte bereit, Schulbuchverlage gestalten didaktisch aufbereitete Texte usw. Private Unternehmen bestimmen dadurch mit, wie Wissen im Unterricht dargestellt wird. Auf diese schulisch, staatlich und gewerblich geschaffenen Vorbedingungen kann die Interaktion im Klassenzimmer immer wieder zurückgreifen, ohne durch sie determiniert zu sein. Die organisatorische Rahmung bietet Gelegenheiten, sich in einer bestimmten Weise zu verhalten: also etwa mit der Autorität der Lehrkraft vor einer Schulklasse zu sprechen oder mit dem Schulbuch oder einem Experiment ein Phänomen zu erläutern.

Um der organisatorischen Rahmung nachzugehen, muss sich die Ethnographie um eine systematische Verknüpfung unterschiedlicher beobachteter Situationen diesseits und jenseits der Schule bemühen. Diese transsituative Perspektive wirft für eine mikrosoziologisch ausgerichtete ethnographische Forschung allerdings Fragen auf. Man müsste den Nachweis erbringen, wie vergangene Situationen für die gegenwärtige Situation wirksam sind, ohne einfach deren Wirksamkeit in der Situation zu behaupten. Wie kann man nun diese Verknüpfung fassen, ohne auf eine Metaphysik des strukturellen Kontexts zurückzugreifen? Die hier vorgeschlagene Lösung lautet, den materiellen Dingen zu folgen, die zwischen den unterschiedlichen Situationen zirkulieren und an der organisatorischen Rahmung des Schulunterrichts beteiligt sind. Dies impliziert eine „multi-sited ethnography“ (Marcus 1998), bei der verschiedene miteinander verknüpfte Felder untersucht und in Bezug zueinander gesetzt werden.

Das hier vorgeschlagene sozio-materielle Verständnis von Bildungsorganisation(en) greift auf die neuere Wissenschaftsforschung zurück (etwa Latour 2001). Materielle Dinge sind hier keine neutralen Werkzeuge, sondern transformieren praktisch und symbolisch, was in einer gegebenen Situation passiert. Einige neuere Studien innerhalb der qualitativen Bildungsforschung zeichnen in dieser Weise die materiellen Bedingungen von Bildung und Erziehung nach (Sørensen 2009; Nohl 2011). Daran anschließend zeige ich im Folgenden, wie diese materiellen Mitspieler verschiedene Situationen (und Organisationen) miteinander verknüpfen, den Unterricht organisatorisch rahmen und damit Ordnung innerhalb der Organisation auf Dauer stellen (siehe hierzu Schatzki 2005). Mit anderen Worten: Um etwas über Bildung zu erfahren, muss die ethnographische Forschung den Schulunterricht und auch die Schule verlassen. Denn vieles, was lokal in der Unterrichtsinteraktion passiert, ist bereits an anderer Stelle vorbereitet worden. Genau hierin liegt die organisatorische Rahmung des Schulunterrichts. Und diese Rahmung wird auch sozio-materiell geleistet. 


\section{Organisatorische Rahmung als sozio-materielle Rahmung}

Wie man sich die sozio-materielle Rahmung vorzustellen hat, möchte ich am Beispiel zweier Merkmale des Schulunterrichts zeigen. Das erste Merkmal betrifft die „Interaktionsordnung“ (Goffman 1983) des Unterrichts; das zweite Merkmal eine spezifische Form der schulischen Wissensvermittlung.

(1) Interaktionsordnung des lehrerzentrierten Schulunterrichts: Im Laufe des 19. Jahrhunderts setzt sich eine uns noch heute vertraute Sozialform des schulischen Unterrichts durch (Hamilton 1990, S. 75f.). Der lehrerzentrierte Zusammenunterricht zielt - im Gegensatz zum älteren Einzelunterricht - darauf ab, dass eine größere Gruppe von Schülern gleichzeitig den Ausführungen einer einzigen Lehrkraft folgt. Instruktive Phasen werden dabei immer wieder von Fragen der Lehrkraft unterbrochen, die von einzelnen Schülern solange beantwortet werden sollen, bis man zu einer richtigen Antwort gelangt. In diesem quasi-sokratischen Dialog mit seiner Abfolge von Lehrerfrage-SchülerantwortLehrerkommentar (Mehan 1979) ist der dritte Zug entscheidend: Durch ihn erhält die Lehrperson immer wieder das Rederecht und die Aufgabe, die Antworten der Schüler zu bewerten und für die gesamte Klasse als falsch oder richtig zu markieren (Kalthoff 1995). Diese Abfolge von Frage-Antwort-Kommentar ist zentraler Bestandteil des lehrerzentrierten Schulunterrichts, der immer noch als die beherrschende Sozialform des Lehrens an deutschen Schulen gilt (Breidenstein 2006, S. 94f.).

(2) Schulische Wissensvermittlung: Der mathematisch-naturwissenschaftliche Schulunterricht setzt ferner auf einen „inszenierte[n] Erkenntnisprozess“ (Erlemann 2004, S. 66). Einerseits steht das Ergebnis der quasi-sokratischen Lehrgespräche und auch der Demonstrationsexperimente bereits fest, andererseits rekonstruieren Schüler unter Anleitung der Lehrerin einen Erkenntnisprozess, dessen Ausgang sie ja - in der Regel - tatsächlich nicht kennen. Und genau auf Grundlage dieser (nur teilweise) gespielten Offenheit interagieren Lehrer und Schüler miteinander. Sie arbeiten dabei gemeinsam an einer disziplinären Sicht, bei der aus vieldeutigen, konkreten Phänomenen oder Sachverhalten relativ eindeutig bestimmte naturwissenschaftliche oder mathematische Objekte werden (ausführlich hierzu Röhl 2013, S. 66-93). Hierzu lenkt die Lehrkraft die Blicke der Schüler auf relevante Merkmale der Experimente bzw. Anschauungsobjekte, übt mit ihnen die Fachsprache ein und transformiert die Beobachtungen schließlich in schriftlicher, generalisierter Form an der Tafel (etwa als vom konkreten Phänomen abstrahierende Formel).

Beide Merkmale des Schulunterrichts können auf eine spezifisch sozio-materielle Form der organisatorischen Rahmung zurückgreifen. Im Fall des ersten Merkmals ist es vor allem das räumliche Arrangement des Klassenzimmers. Hier zeichne ich im Folgenden nach, wie sich diese Form der sozio-materiellen Rahmung als schulenübergreifende Gattung geschichtlich herausgebildet hat (2.1). Wir bleiben im Feld der Schule, verlassen aber das konkrete Klassenzimmer der ethnographischen Gegenwart, um zu dessen geschichtlichen Ursprüngen zu 
gelangen. Beim zweiten Merkmal ist es die spezifische Gestalt didaktischer Artefakte, die rahmend wirkt. In diesem Fall folge ich den Lehrmitteln zu ihrem individuellen Ursprung, also zu den Stätten ihrer Konstruktion in der Lehrmittelindustrie (2.2). Damit verlassen wir die Schule und gehen der „kulturellen Biographie der Dinge“ (Kopytoff 1986) nach, innerhalb derer sich der Status der Dinge mehrfach ändert. Wichtig sind in diesem Zusammenhang vermittelnde Instanzen, die zwischen Lehrmittelindustrie und Schulunterricht stehen (2.3).

\subsection{Unterrichtsräume und die Interaktionsordnung des Schulunterrichts}

Ein Blick in die Geschichte der Unterrichtsräume zeigt, dass die zunehmende Verbreitung des Zusammenunterrichtens mit dem Siegeszug eines neuen, spezifisch für diesen Zweck geschaffenen räumlichen Arrangements zusammenfällt.

In der frühen Neuzeit dienten noch einfache Wohnhäuser und Wohnstuben als Orte des Unterrichts (Schmidt 1967, S. 520-530). Unterricht war vielerorts einklassig und fand in einem einzelnen Raum innerhalb des Schulhauses statt (Lange 1967, S. 48-81). Verschiedene räumliche Arrangements existierten dabei nebeneinander: In den deutschen Lese- und Schreibschulen der frühen Neuzeit waren die Bänke der Schüler nicht allesamt Richtung Lehrerpult ausgerichtet, sondern in Reihen entlang der Wände. Die Lateinschulen unterschieden die Schüler gemäß ihren Kenntnissen und teilten sie in entsprechende, im Raum verteilte Gruppen ein, die gemeinsam arbeiten bzw. beim Schulmeister vorsprechen mussten (Schiffler/Winkeler 1994, S. 60ff.). In den weltlichen Rechenschulen waren die Schüler hingegen um einen Tisch herum angeordnet, auf dem sie unter Anleitung des Rechenmeisters und mit Hilfe eines Rechenbretts selbständig Aufgaben rechnen mussten (Göhlich 2009, S. 93f.). Eine einheitliche Ausrichtung aller Schüler im Raum gab es nicht. Schulischer Unterricht war in der frühen Neuzeit mehrheitlich selbsttätiges Studium mit dem Ziel präzisen Memorierens und Repetierens der Lerninhalte (Petrat 1979, S. 37-47). Dies konnte in Form des Einzelunterrichts oder aber gruppenweise geschehen. Der frühneuzeitliche Schulraum war dementsprechend nicht der Raum zur gleichzeitigen Unterrichtung einer relativ homogenen Klasse durch eine Lehrperson, sondern „der Übungsraum einer gegliederten Klasse“ (Lange 1967, S. 111; Hervorhebung T.R.).

Am Ende des 18. Jahrhunderts wurde Bildung mehr und mehr zur Aufgabe des Staates (Petrat 1979) und staatliche Institutionen griffen zunehmend mit Verordnungen auch in den Bau der Schulen und die Ausstattung der Klassenzimmer ein (Schmidt 1967, S. 530-550). Mit der sich langsam durchsetzenden Schulpflicht entstand so auch ein neuer Typus Schulgebäude. Im 19. Jahrhundert setzten sich in den Städten große kasernenartige Schulgebäude durch, die jeder nach Jahrgang eingeteilten Klasse einen Raum zuwiesen (Göhlich 1993, S. 311). Durch die Aufteilung der Schüler nach Jahrgängen erhoffte man sich, eine große Zahl von Schülern unterrichten und gleichzeitig den unterschiedlichen Begabungen gerecht wer- 
den zu können, da man davon ausging, es mit relativ homogenen Gruppen zu tun zu haben (Lange 1967, S. 295). Innerhalb dieser „wilhelminischen Schulkaserne[n]“ (Göhlich 1993, S. 311) reihte sich in großen Korridoren Klassenzimmer an Klassenzimmer. Die Schüler saßen an feststehenden und in Reihen angeordneten Schulbänken, die eine aufrechte Haltung abverlangten und allesamt Richtung Stirnwand zeigten (Hnilica 2010, S. 155-159). Der Blick der Schüler war dadurch auf das durch ein Podium erhöhte Lehrerpult und eine zentrale Wandtafel gerichtet.

Dieser kurze geschichtliche Abriss zeigt, wie die Unterrichtsinteraktion auf eine ihr vorgängige Rahmung aufbauen kann. Mit der zunehmenden Verstaatlichung der Bildung kristallisiert sich auch eine Ausdifferenzierung und Spezifizierung schulischer Gebäude und Unterrichtsräume heraus. Durch Verordnungen und Baunormen wirken staatliche Behörden nicht nur administrativ, sondern - vermittelt über die Schularchitektur - auch in die Interaktionsordnung des Unterrichts selbst hinein. Dies zeigt sich in mehrfacher Hinsicht:

(1) Abgrenzung nach Außen: Durch die Separierung der Unterrichtsteilnehmer in geschlossenen Räumen und die Abgrenzung nach Außen ist der Unterricht der alltäglichen Lebenswelt enthoben und vor Störungen geschützt. Dies erleichtert zum einen die Durchsetzung einer Interaktionsordnung, die anderen Regeln als denen der Alltagsinteraktion folgt (s.u.). Zum anderen wird dadurch die relativ ablenkungsfreie Fokussierung auf schulische Themen begünstigt. Ferner liegt hierin die von der Organisationsforschung beobachtete Intransparenz des unterrichtlichen Interaktionssystems (etwa bei Drepper/Tacke 2012, S. 224ff.) ganz praktisch-materiell in den Wänden und Türen der Klassenzimmer begründet. Dies entlastet zum einen andere Bereiche der Organisation, zum anderen erlaubt es jenseits formaler Regularien „pädagogische Permissivität“ (Wernet 2003). Für die Schule als Organisation zählen nur ausgewählte Aspekte der Unterrichtsinteraktion - etwa die Bewertung der Schüler in Form von Zensuren. Und Schüler und Lehrer wiederum können formale Vorgaben ohne ständige Überwachung relativ frei adaptieren oder sich diesen gar widersetzen.

(2) „Die Kunst der Verteilungen“ (Foucault 1994, S. 181-191): Die modernen Schulgebäude unterteilen Schüler in nach Altersjahrgängen unterteilte Gruppen (Hnilica 2010, S. 158f.) Einerseits soll so die relative Homogenität der zu prozessuierenden Gruppe gewährleistet werden, um so Kontingenz in der unterrichtlichen Interaktion zu minimieren. Andererseits kann dies auch als eine Technik der Disziplinierung verstanden werden. Eine überschaubare Gruppe von Schülern ist innerhalb des Klassenzimmers auf individualisierte Plätze verwiesen. An ihren Plätzen sind die Schülerkörper in sitzender Position ruhig gestellt, vereinzelt und dadurch durch eine einzelne Lehrperson handhabbar.

(3) „Zentralperspektivisches“ (Göhlich 1993, S. 311) Arrangement: Das moderne Klassenzimmer richtet eine große Zahl von Schülern auf einen Bereich aus, der von einem einzelnen, ihnen gegenübergestellten Lehrer besetzt ist. Dies erleichtert die um die 
Lehrerin zentrierte Abfolge von Frage-Antwort-Kommentar, die an eine Gruppe von Menschen gerichtet ist. Gleichzeitig kann die Lehrkraft die gesamte Klasse leicht im Blick behalten und die Aufrechthaltung eben dieser Interaktionsordnung kontrollieren.

Das räumliche Arrangement des frontal ausgerichteten Klassenzimmers sorgt also in mehrfacher Hinsicht dafür, dass die Organisation Schule ihre Ziele verfolgen kann. Das Klassenzimmer isoliert und homogenisiert eine Klasse, in der die einzelnen Schüler zugleich an ihren Plätzen individualisiert sind. Zugleich richtet es die individualisierten Körper in spezifischer Weise aus und unterstellt sie der Kontrolle durch den Lehrerblick. Damit wird eine bestimmte Form unterrichtlicher Interaktion wahrscheinlicher, nämlich der lehrerzentrierte Zusammenunterricht (vgl. Hausendorf 2008).

\subsection{Lehrmittel und Disziplinierung}

Kommen wir zum zweiten Fall, an dem die These von der sozio-materiellen Rahmung des Schulunterrichts plausibilisiert werden soll: die sogenannten Lehrmittel und die durch sie geleistete Disziplinierung von Schülern. Insbesondere in den naturwissenschaftlichen Fächern finden sich eine Reihe von experimentellen Arrangements und technischen Gerätschaften, mit deren Hilfe schulisch relevantes Wissen für die Schüler anschaulich gemacht werden soll. Am Beispiel eines Schülerexperimentierkastens für den Physikunterricht wird deutlich, dass Lehrmittel dabei in zweifacher Hinsicht Disziplin einfordern (vgl. Abb. 1): Zum einen erleichtern sie eine disziplinäre (d.h. fachliche) Sicht auf die Dinge, zum anderen wirken sie disziplinierend. Im einen Fall geht es um Disziplin im Sinne einer Wissensordnung, im anderen Fall um eine Verhaltensordnung (vgl. Künzli 2008). 


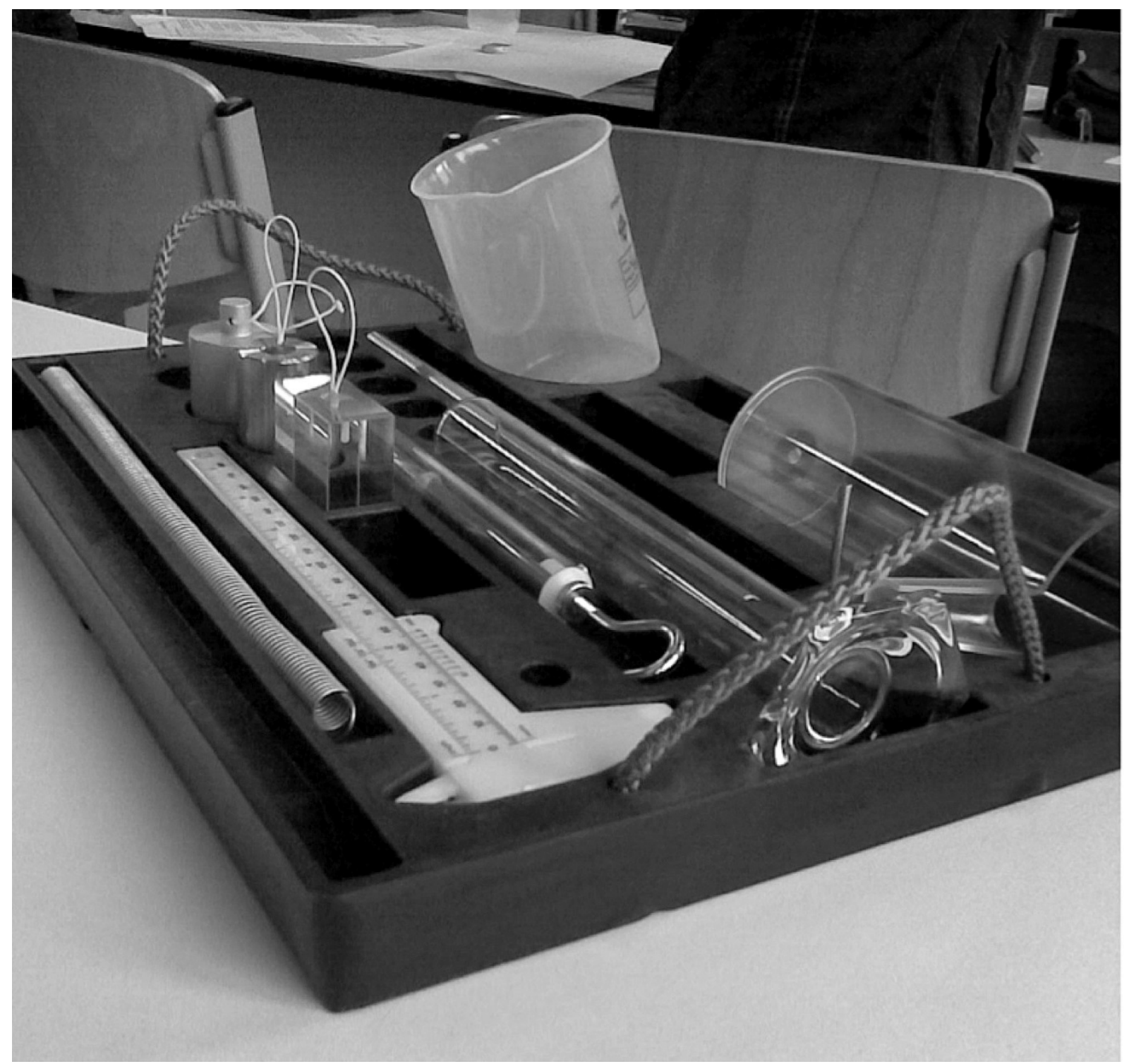

Abb. 1: Schülerexperimentierkasten des Herstellers MEKRUPHY (eigene Fotografie)

(1) Didaktische Reduktion: Der rund $40 \times 30 \mathrm{~cm}$ große Kasten bzw. seine Einlage besteht aus Kunststoff und ist in einem einfachen matten Schwarz gehalten. Vor diesem unauffälligen Hintergrund heben sich die unterschiedlichen Gegenstände in seinem Inneren umso deutlicher hervor. Zugleich sind sie in eigens für sie geschaffenen Mulden noch einmal individualisiert. Der funktional gestaltete Kasten leistet so zunächst eine Fokussierung des Blicks und des Griffs auf seine wesentlichen Bestandteile. Mit Hilfe dieser Bestandteile können die Schüler unter Anleitung der Lehrperson und eines dazugehörigen Aufgabenhefts eine Reihe von unterschiedlichen Experimenten zur Mechanik durchführen. Die einzelnen Gegenstände folgen wiederum einem geradlinigen Gestaltungsprinzip. Statt gestalterischer Schnörkel finden sich auf relevante Merkmale reduzierte Gegenstände. So sind etwa mehrere Gewichte enthalten, die dazu dienen, den Schülern die Messung der Gewichtskraft nahezubringen. Diese entsprechen in ihrer Aufmachung typischen Gewichten, wie man sie etwa von Kaufmannswaagen kennt. Ferner stützen sich die Gegenstände innerhalb 
des Kastens wechselseitig in ihrer Bedeutung als didaktisch-physikalische Objekte. Eine Feder könnte auch Bestandteil eines Spielzeugbaukastens sein, innerhalb des Experimentierkastens ist seine Bedeutung als physikalisches Lehrmittel aber relativ klar.

(2) Kontrollfunktion: Eine weitere Eigenschaft des Kastens macht ihn außerdem auch zu einem Kontrollinstrument. Durch die individuellen Mulden, die jedem Gegenstand einen spezifischen Platz im Kasten zuweisen, kann die Lehrperson am Ende des Schülerexperiments auf den ersten Blick sehen, ob der Kasten vollständig zurückgegeben wurde. Der Kasten erleichtert so die Kontrolle der Schüler durch eine einzelne Lehrkraft.

An diesem kurzen Beispiel zeigt sich, dass in der Lehrmittelindustrie Vorkehrungen getroffen werden, um antizipierten Anforderungen des Schulunterrichts entgegenzukommen. Die sozialwissenschaftliche Forschung zu Technik und Design hat darauf hingewiesen, dass bereits früh in der Entwicklung technischer Artefakte die zukünftigen Nutzer und der praktische Gebrauch antizipiert werden (etwa Rammert u. a. 1998; Julier 2012). Bei der Entwicklung gilt es verschiedene Nutzungsszenarien gegeneinander abzuwägen und mit anderen Überlegungen (z.B. Kosten des Materials) in Einklang zu bringen (MacKenzie/Wajcman 1999). Für die Lehrmittelindustrie finden sich bislang kaum Studien, die sich mit diesen Entwicklungsprozessen beschäftigen (siehe aber Wiesemann/Lange 2015). Hier steht noch genauere ethnographische Forschung aus, die systematisch ergründet, wie diese Aushandlungsprozesse auf Seiten der Lehrmittelindustrie ablaufen: Welche Abteilungen und Akteure sind an der Entwicklung beteiligt? Welche Kriterien werden von wem in Anschlag gebracht und erfolgreich umgesetzt? Welches Modell vom Unterrichten haben die Entwickler? Usw.

Auf Grundlage der hier vorgestellten Überlegungen zur Gestalt der Lehrmittel lässt sich festhalten, dass die Hersteller eine fachliche Sicht und einen schonenden Gebrauch der didaktischen Artefakte vorbereiten. Besonders deutlich wird die Relevanz dieser didaktischen Reduktion und pädagogischen Stabilisierung dann, wenn Lehrer Gegenstände im Unterricht einsetzen, die nicht eigens für didaktische Zwecke geschaffen wurden. Als beispielsweise einmal ein Lehrer mehrere Packen Kopierpapier in den von mir beobachteten Unterricht mitbrachte, fragte eine Schülerin erschreckt, ob die Klasse jetzt einen Test schreibe. Der Lehrer musste sie deshalb mit dem Hinweis beruhigen, dass er damit nur "etwas zeigen“ wolle. Bei den didaktisch gestalteten Lehrmitteln ist eine solche Reaktion seitens der Schüler hingegen eher unwahrscheinlich. Zwar ist ihnen oft unklar, was mit einem fraglichen Gegenstand gezeigt werden soll, dass etwas mit einem Lehrmittel gezeigt werden soll, wird nicht in Frage gestellt. Im Gegenteil: Für die Schüler signalisiert ein sichtbar getragenes oder auf dem Pult platziertes Lehrmittel ein bevorstehendes Experiment und verheißt damit zumindest eine Abwechslung vom „interaktionalen Gleichfluss“ (Krummheuer 2002, S. 46) des Unterrichtgeschehens. 


\subsection{Die Einschulung der Dinge}

Zwischen Lehrmittelherstellern und Schulunterricht vermitteln verschiedene Instanzen, die dafür sorgen, dass aus einem zu verkaufenden Produkt (Herstellersicht) ein didaktisches Werkzeug (Lehrersicht) wird. Nachdem ein Lehrmittel produziert wurde, muss zunächst einmal ein Bedarf geschaffen und die Kundschaft für das Produkt gewonnen werden. Hier kommt dem Marketing eine entscheidende Rolle zu. Das Marketing ist dabei nicht als Beiwerk fertiger Produkte zu verstehen, sondern als eine eigenständige Leistung, durch die überhaupt erst begehrenswerte Konsumgüter und die dazugehörigen Käufer entstehen (Cochoy 1998). Die Lehrmittelindustrie leistet dies etwa über die zahlreichen Prospekte und Kataloge, in denen auf die didaktischen Vorzüge und pädagogische Eignung der angebotenen Waren hingewiesen wird. Auf Messen (etwa der „Didacta“) und Lehrerfortbildungen stellen die Hersteller ferner Neuheiten vor, führen in deren Gebrauch ein und versuchen praktisch und diskursiv die Lehrkräfte von ihren Produkten zu überzeugen. Diese Arbeit am Produkt möchte ich kurz am Beispiel der interaktiven Whiteboards illustrieren. ${ }^{2}$

Besonders an der Bewerbung neuer Unterrichtsmedien wie dem interaktiven Whiteboard wird deutlich, wie sich die Lehrmittelindustrie ihre Kundschaft vorstellt und welche Wünsche bzw. Ängste sie ihr zuschreibt. Zum einen stellen die Prospekte die interaktiven Whiteboards als Wegbereiter eines neuen, besseren Unterrichts dar. Der Hersteller "SMART“ spricht beispielsweise von "spannenden Unterrichtswerkzeugen“ und verheißt „begeisterte Schüler“ sowie ein "packendes Erlebnis“. Beim Konkurrenten „Promethean“ wird das Klassenzimmer zum Ort, "where we unleash the potential of every student and every teacher every day“. Schüler werden zu "Stars“ und man verspricht einen „lebendigen“ Unterricht. Die unterschiedlichen Hersteller begründen diese Attraktivität der interaktiven Whiteboards mit der Möglichkeit, interaktive Multimediainhalte in den Unterricht einbinden zu können. Dabei stellt die Werbung die Whiteboards vor allem der konventionellen Wandtafel gegenüber, die als veraltetes und langweiliges Medium gilt. Eine neue Epoche verkündend, spricht beispielsweise das Unternehmen „Legamaster" vom „Ende der Kreidezeit”.

Neben der Hoffnung auf einen besseren, lebendigeren Unterricht adressiert das Marketing zum anderen aber auch unterschiedliche Ängste. Dies betrifft etwa die Befürchtung, der technischen Handhabung nicht gewachsen zu sein. Dementsprechend betonen die Hersteller in den Prospekten die „kinderleichte Handhabung“ („Legamaster“) oder die „,intuitive, einfache Bedienbarkeit der Produkte“ („SMART“). Begründet wird dies damit, dass sich das Design der interaktiven Whiteboards an ältere Unterrichtsmedien anlehnt. So werden einige

\footnotetext{
' Die Formulierung „Einschulung der Dinge“ entlehne ich Wiesemann und Lange (2015). 2Interaktive Whiteboards verbinden eine berührungsempfindliche Projektionsfläche mit einem Kurzdistanzbeamer, an den ein Computer angeschlossen werden kann (Schlieszeit 2010). Anders als bei gängigen Computerpräsentationen kann man - je nach Modell - mit Finger oder speziellen "Stiften“ direkt auf der Projektionsfläche schreiben und Programme bedienen. Mit dem interaktiven Whiteboard wird die Tafel gewissermaßen digital: interaktive Elemente können eingebunden und das ,Tafelbild' abgespeichert werden.
} 
Modelle beispielsweise mit einem stiftähnlichen Gegenstand bedient und ,beschriftet'. Die Hersteller stellen diesen Umstand in ihren Prospekten klar heraus: „Vorteil 4: Stift als bekanntes Schreibinstrument“ („Legamaster“); „Der Stift ist der Stift, der Schwamm ist der Schwamm“ („SMART“). Ferner begegnet das Marketing einer Angst vor Vandalismus. Immer wieder ist die Rede davon, wie robust die Geräte sind. Um dies zu belegen, schlagen Vertreter der Hersteller demonstrativ auf die Projektionsflächen ein und weisen auf die Langlebigkeit des Produkts hin. Der Hersteller „Legamaster“ formuliert dies in einem Prospekt so:

„Die Gefahr von Schäden durch Vandalismus ist nahezu ausgeschlossen - durch eine emaillierte, robuste und kratzfeste Metalloberfläche mit 25 Jahren Garantie. Damit sind unsere qualitativ hochwertigen Projektionsflächen dauerhaft einsetzbar, denn sie wurden für den strapazierfähigen Einsatz konzipiert."

Damit übernimmt das Marketing eine wichtige Funktion innerhalb der schrittweisen schulischen Aneignung der Lehrmittel und Unterrichtsmedien. Es werden begehrenswerte Produkte geschaffen, die sowohl auf (antizipierte) Wünsche als auch Ängste der Kundschaft der Lehrkräfte reagieren. Für die organisatorische Rahmung ist dies wichtig, da dem Marketing damit eine wichtige Funktion zukommt. Hier entscheidet sich, welche Produkte gegenüber der Kundschaft erfolgreich vermarktet und letztlich verkauft werden können. Und mit diesen Produkten betreten nach Verkaufsabschluss auch irgendwann bestimmte Formen der schulischen Wissensvermittlung den Unterricht (siehe 2.2).

Am Beispiel naturwissenschaftlicher Lehrmittel (Experimentierkästen, Demonstrationsmessgeräte usw.) zeige ich nun, was passiert, wenn die didaktischen Produkte erfolgreich an Schulen verkauft werden. Die Schule als Organisation leistet hier zweierlei: Sie stellt einerseits eigene Räumlichkeiten („Sammlungen“) zur Aufbewahrung, Inventarisierung und Vorbereitung von Experimenten und anderen Lehrmitteln bereit. Und andererseits werden einzelne Fachlehrer („Sammlungsleiter/in“) mit der Beschaffung und Verwaltung dieser Lehrmittel betraut.

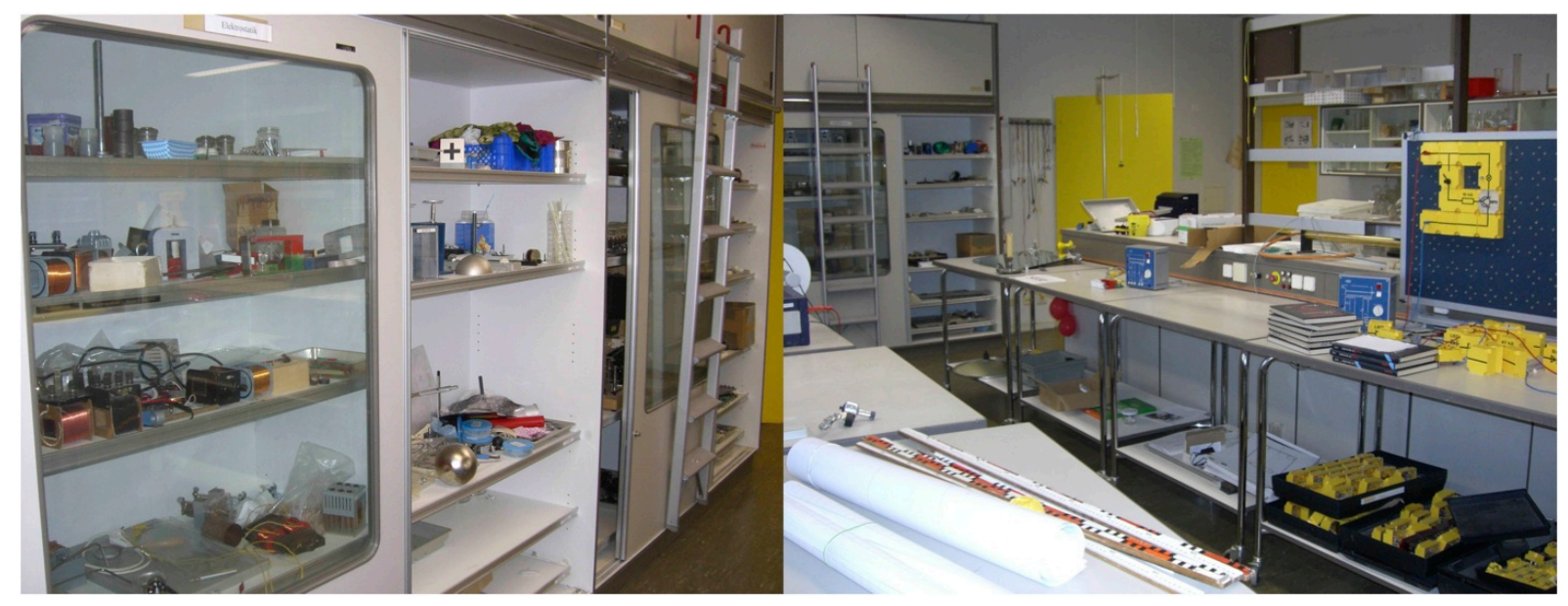

Abb. 2: Eine physikalische Sammlung (eigene Fotografie) 
In den Sammlungen findet sich ein Sammelsurium an Dingen, mit denen naturwissenschaftliches Wissen an die Schüler vermittelt werden soll (siehe Abb. 2). In Regalen und mit Glastüren versehenen Schränken ist eine Reihe von Dingen aufbewahrt, mit deren Hilfe sich verschiedene Experimente durchführen lassen. Einzelne Fachlehrer sind als "Sammlungsleiter" zuständig für die Sammlung. Sie inventarisieren und verwalten den schulischen Bestand an „Experimenten“ und schaffen gegebenenfalls neue Geräte und Versuche durch Bestellung bei spezialisierten Herstellern an. Trifft ein neues „Experiment“ in der Sammlung an, so wird es zunächst vom Sammlungsleiter auf seine Funktionsfähigkeit hin geprüft und hinsichtlich seiner didaktischen Eignung bewertet. An einer von mir beforschten Schule konnte ich den Leiter der Physiksammlung bei seiner Arbeit begleiten und beobachten:

Herr Baier führt mich zu einem beachtlichen Stapel von Blättern, auf denen bereits einige Geräte erfasst sind. Darauf finden sich Angaben wie Gerätebezeichnung, Kaufdatum, Rechnungsnummer, Preis, aber auch Themengebiet und Aufbewahrungsort in der Sammlung etc. Wir gehen wieder zurück zum Rollwagen. Der Lehrer öffnet nun das kleine Paket, das er anfangs mitgebracht hat. Stolz zeigt er mir den Inhalt des Pakets. Es handelt sich um zwei stabartige Gegenstände, an deren Ende eine Kugel bzw. ein Ring aus Messing befestigt sind. Er führt mir vor, dass die Kugel mit Leichtigkeit durch den Ring hindurch passt. Wenn man aber die Kugel erhitze, so wird deutlich, dass dies nicht mehr gehe. Dieses „schöne neue Lehrexperiment" kann er nun aufnehmen, dann sei es „Teil der Physiksammlung“. Er packt es wieder in den Karton hinein und beschriftet diesen mit einem schwarzen Filzstift „Kugel + Ring 29.3.2011“. Den Karton legt er zunächst zur Seite, um ihn dann später in den Schrank mit der Aufschrift „Wärmelehre“ einzuräumen und mittels eines Aufklebers mit einer Inventurnummer zu versehen.

Der Sammlungsleiter bewertet am „Lehrexperiment“ zunächst zweierlei. Einerseits prüft er als verantwortlicher Vertreter der Organisation, ob das gekaufte Produkt korrekt und ohne Beanstandungen geliefert wurde. Andererseits hat er als Fachlehrer den Unterricht im Blick und beurteilt die fachdidaktische Eignung des Lehrmittels. Ein wichtiges Kriterium ist dabei, dass an den Dingen etwas deutlich wird. Mit der anschließenden Inventarisierung reiht der Sammlungsleiter die Dinge in eine administrative Ordnung ein und macht sie z.B. innerhalb von Rechenschaftsberichten verfügbar. Aus Sicht der Organisation Schule existieren die Dinge anhand eines listenförmigen Inventars, das Auskunft über einen Bestand didaktischer Hilfsmittel gibt. Hiermit kann die Schule Rechenschaft über Ausgaben und deren Legitimität ablegen. Wenn der Sammlungsleiter das "schöne neue Lehrexperiment“ in den Schrank mit der Aufschrift „Wärmelehre“ einräumt, erhält es aber auch einen Platz innerhalb einer curricularen Ordnung von Themen. Die meisten der Regale und Schränke in der Sammlung sind in einer solchen Weise einzelnen curricularen Themen zugeordnet und weisen dies auch sichtbar durch entsprechende Beschriftungen („Mechanik 1“, „Elektrostatik 2“) aus. Nimmt man die Perspektive der Fachlehrer ein, sieht man anhand der schriftlich festgehaltenen In- 
ventarisierung auch, welche curricularen Themengebiete durch welche Experimente abgedeckt sind und wo sie jeweils zu finden sind.

Angesichts der Vielzahl an Dingen innerhalb der Sammlung gewährt nur die schriftliche Registrierung und Inventarisierung, dass die Organisation und ihre Mitglieder über die Dinge verfügen. Aus einer bloßen Ansammlung von Dingen wird eine geordnete Sammlung. Die Inventurnummer erfüllt hier eine wichtige Funktion: Mit ihr wird das konkrete Ding mit der administrativen Ordnung verbunden. Sie sichert die Referenz zwischen dem Ding in Schrank und dem in der Liste und damit die Verbindung von curricularer und administrativer Ordnung. Um die Rolle des Einordnens auf den Punkt zu bringen: Das Einordnen der Dinge in Schränke und Listen ist zugleich ihre Geburtsstunde als schulische Objekte, sei es als curricular bestimmtes „Lehrexperiment“ oder als administrativ bestimmter Bestand innerhalb einer Sammlung. Zum „Teil der Sammlung“ wird er es erst dadurch, dass es in zweifacher Art und Weise durch den Sammlungsleiter angeeignet wird.

Mit der Einordnung in den Sammlungen ist eine weitere Station in der Biographie schulischer Lehrmittel angesprochen. Aus einem begehrenswerten Produkt wird nun administrativ erfasstes Eigentum (Sicht der Schulverwaltung) und ein curricular bestimmtes didaktisches Werkzeug, das Möglichkeiten eröffnet, Unterricht zu gestalten (Sicht der Lehrkräfte). Neben dieser ordnenden Funktion erfüllen die Sammlungen aber auch unterrichtsvorbereitende Aufgaben. Hier können die Lehrer ungestört den Einsatz der Lehrmittel in Form der Experimente einüben, die Schüler sind systematisch von den Sammlungen ausgeschlossen. Ziel ist der möglichst reibungslose Ablauf und die Sichtbarkeit der Phänomene. Wenn die Lehrmittel den Unterricht betreten, soll gewährleistet sein, dass sie gemäß ihrer in der Lehrmittelindustrie eingeschriebenen Funktion ihren Dienst verrichten. Die Sammlung als abgeschlossener Ort innerhalb der Organisation ermöglicht so einerseits die relativ störungsfreie Umsetzung eines in der Lehrmittelindustrie erdachten didaktischen Programms. Andererseits eignen sich hier die einzelnen Lehrkräfte die Lehrmittel an, nehmen Anpassungen vor und tüfteln an den experimentellen Arrangements herum, bis sie meinen, dass das Experiment den Ansprüchen ,ihres' Unterrichts genügt. Mit der Vorbereitung der Experimente in der Sammlung greifen sie vorweg, was später im Unterricht passieren soll.

\section{Abschließende Bemerkungen}

Schulunterricht ist keine voraussetzungslose Interaktion, sondern findet innerhalb eines organisatorischen Rahmens statt. Dies wurde anhand verschiedener Beispiele (Unterrichtsräume, Lehrmittel und ihre Biographie) als sozio-materielle Rahmung gefasst. Der Zugang zu dieser sozio-materiellen Rahmung kann auf verschiedene Weise erfolgen. Anders als et-

\footnotetext{
3. Für Museen und ihre Ausstellungsstücke konnten Doering und Hirschauer (1997) ähnliche, sich
} überlagernde Ordnungssysteme ausmachen. 
wa Scheins Modell der Organisationskultur (1984) gehe ich davon aus, dass der Beitrag der Dinge zur Praxis trotz ihrer Sichtbarkeit nur schwer zu fassen ist. Sie sind mehr als bloß kulturelle Symbole, sondern praktisch an Bildung und anderen Feldern beteiligt. Aber diese praktische Dimension muss erst einmal expliziert werden und ist nicht ohne weiteres an den Artefakten abzulesen (vgl. Hirschauer 2001). Die Ethnographie ist deshalb darauf angewiesen, auf verschiedene methodische Werkzeuge zurückzugreifen. Hier wurde dies mit Hilfe historischer Vergleiche, der Beschäftigung mit der Gestalt(ung) der Dinge sowie der Metapher der „Biographie“ der Dinge erreicht. Allen Zugängen gemein ist die (gedankliche oder tatsächliche) Mobilisierung des ethnographischen Beobachters, der nun nicht mehr im Klassenzimmer und auch nicht in der Schule allein verweilen kann. Die Umsetzung einer transsituativen Ethnographie der Bildung müsste all die Orte besuchen, die mittelbar an Schulunterricht beteiligt sind. Hier habe ich erste Analysen in diese Richtung vorangetrieben. Weitere Untersuchungen (etwa der Entwicklungsprozesse in der Lehrmittelindustrie) müssten folgen und an diese Überlegungen anschließen.

Was konnte mit den ersten Analysen gezeigt werden? Über die Lehrmittel und Unterrichtsräume werden den Schülern Haltungen und Handlungen abverlangt, die in unhinterfragter Selbstverständlichkeit zum alltäglichen Treiben („daily grind“; Jackson 1968, S. 1-37) im Klassenzimmer dazugehören. Die Organisation Schule sorgt deshalb mit den Dingen und Räumen nicht nur dafür, dass ihre Mitglieder etwas lernen, sondern auch dafür, dass ein relativ stabiles wiederkehrendes Interaktionsgeschehen zustande kommt und auf Dauer gestellt wird (vgl. Schatzki 2005). Indem die Unterrichtsteilnehmer sich der sozio-materiellen Rahmung fügen, bestätigen sie die schulische Organisation der Wissensvermittlung. Diese setzt vermittelt über die Dinge (unter anderem) auf:

(1) eine Asymmetrie und Hierarchie zwischen Lehrern und Schülern (Unterrichtsräume, Kontrollfunktion der Lehrmittel, Ausschluss der Schüler aus den Sammlungen);

(2) eine fachliche und curriculare Sequenzierung des Wissens, in der Dinge für Schulstunden in Fächern und für die Abfolge von Themen im Lehrplan stehen („ein Demonstrationsexperiment zur Mechanik im Fach Physik“) (curriculare Ordnung der Lehrmittel in der Sammlung);

(3) eine disziplinäre Sicht, bei der Dinge und Phänomene in schulfachlich relevanter Weise betrachtet werden sollen (didaktische Reduktion der Lehrmittel).

Dinge sind damit in (mindestens) dreierlei Hinsicht vermittelnde Instanzen der Organisation Schule: Sie stehen für unterschiedliche Positionen in einer Interaktionsordnung, sie legen

' Für Schein sind Artefakte kulturelle Symbole, die auf einen bestimmten Bereich einer Organisationskultur verweisen: etwa der Chefsessel, der die Position eines Managers symbolisiert. Als solche liegen sie - im Gegensatz zu den kulturellen Vorstellungen der Organisationsmitglieder - offen an der Oberfläche der Organisationskultur. Damit verkennt dieses Modell aber, dass der praktische Beitrag der Artefakte zu unseren alltäglichen Aktivitäten (etwa in Organisationen) nicht immer offensichtlich ist und sich dem reflektierten Nachdenken entzieht (vgl. Hörning 2005). 
zeitlich-thematische Abfolgen nahe und sie fordern unterschiedliche Zugänge zur Welt ein. Mit der Verschiebung des ethnographischen Blicks zu dieser sozio-materiellen Dimension des Schulunterrichts ist dementsprechend zugleich auch die Chance verbunden, institutionalistische und organisatorische Fragen stärker einzubeziehen, ohne von der Idee der unmittelbaren Beobachtbarkeit abzuweichen (siehe hierzu auch Schmidt/Volbers 2011). Das Ziel bleibt ein genaues Verfolgen und Nachzeichnen kultureller Praktiken, nur dass sich diese eben auch vermittels der Dinge auffinden lassen.

\section{Literatur}

Breidenstein, G. (2006): Teilnahme am Unterricht: Ethnographische Studien zum Schülerjob. Wiesbaden: VS.

Cochoy, F. (1998): Another Discipline for the Market Economy. Marketing as Performative Knowledge and Know-how for Capitalism. In: Callon, M. (Hrsg.): The Laws of the Markets. Oxford: Blackwell, S. 194-221.

Corsaro, W. A. (1985): Friendship and Peer Culture in the Early Years. Norwood: Ablex.

Crozier, M. (1964): The Bureaucratic Phenomenon. Chicago: University of Chicago Press.

Doering, H./Hirschauer, S. (1997): Die Biographie der Dinge. Eine Ethnographie musealer Repräsentationen. In: Hirschauer, S. / Amann, K. (Hrsg.): Die Befremdung der eigenen Kultur. Frankfurt am Main: Suhrkamp, S. 267-297.

Drepper, T. / Tacke, V. (2012): Die Schule als Organisation. In: Apelt, M./ Tacke, V. (Hrsg.): Handbuch Organisationstypen. Wiesbaden: VS, S. 205-237.

Eberle, T.S./Maeder, C. (2011): Organizational Ethnography. In: Silverman, D. (Hrsg.): Qualitative Research. Issues of Theory, Method and Practice. London: Sage, S. 53-73.

Erlemann, M. (2004): Inszenierte Erkenntnis. Zur Wissenschaftskultur der Physik im universitären Lehrkontext. In: Arnold, M. / Fischer, R. (Hrsg.): Disziplinierungen. Kulturen der Wissenschaften im Vergleich. Wien: Turia + Kant, S. 53-90.

Foucault, M. (1994): Überwachen und Strafen. Die Geburt des Gefängnisses. Frankfurt am Main: Suhrkamp.

Goffman, E. (1983): The Interaction Order: American Sociological Association, 1982 Presidential Address. In: American Sociological Review 48, S. 1-17.

Göhlich, M. (1993): Die pädagogische Umgebung. Eine Geschichte des Schulraums seit dem Mittelalter. Weinheim: Deutscher Studien Verlag.

Göhlich, M. (2009): Schulraum und Schulentwicklung. Ein historischer Abriss. In: Böhme, J. (Hrsg.): Schularchitektur im interdisziplinären Diskurs. Territorialisierungskrise und Gestaltungsperspektiven des schulischen Bildungsraums. Wiesbaden: VS, S. 89-102.

Hamilton, D. (1990): Learning about Education. An Unfinished Curriculum. Buckingham: Open University Press.

Hausendorf, H. (2008): Interaktion im Klassenzimmer. Zur Soziolinguistik einer riskanten Kommunikationspraxis. In: Willems, H. (Hrsg.): Lehr(er)buch Soziologie. Für die pädagogischen und soziologischen Studiengänge. Wiesbaden: VS, S. 931-957.

Hirschauer, S. (2001): Ethnografisches Schreiben und die Schweigsamkeit des Sozialen. In: Zeitschrift für Soziologie 30, S. 429-451.

Hnilica, S. (2010): Schulbank und Klassenzimmer. Disziplinierung durch Architektur. In: Egger, R. / Hackl, B. (Hrsg.): Sinnliche Bildung? Pädagogische Prozesse zwischen vorprädikativer Situierung und reflexivem Anspruch. Wiesbaden: VS, S. 141-162.

Hörning, K.H. (2005): Lob der Praxis. Praktisches Wissen im Spannungsfeld zwischen technischen und sozialen Uneindeutigkeiten. In: Gamm, G./Hetzel, A. (Hrsg.): Unbestimmtheitssignaturen der Technik. Eine neue Deutung der technisierten Welt. Bielefeld: Transcript, S. 297-310.

Jackson, P.W. (1968): Life in Classrooms. New York: Holt.

Julier, G. (2012): Design als Praxis. Eine praxistheoretische Perspektive. In: Moebius, S. / Prinz, S. (Hrsg.): Das Design der Gesellschaft. Zur Kultursoziologie des Designs. 
Bielefeld: Transcript, S. 227-243.

Kalthoff, H. (1995): Die Erzeugung von Wissen. Die Fabrikation von Antworten im Schulunterricht. In: Zeitschrift für Pädagogik 41, S. 925-939.

Kalthoff, H. (1996): Das Zensurenpanoptikum. Eine ethnographische Studie zur schulischen Bewertungspraxis. In: Zeitschrift für Soziologie 25, S. 106-124.

Kelle, H. (1999): Geschlechterterritorien. Eine ethnographische Studie über die Spiele neunbis zwölfjähriger Schulkinder. In: Zeitschrift für Erziehungswissenschaft 2, S. 211228.

Kopytoff, I. (1986): The Cultural Biography of Things: Commoditization as Process. In: Appadurai, A. (Hrsg.): The Social Life of Things. Commodities in Cultural Perspective. Cambridge: Cambridge University Press, S. 64-94.

Krummheuer, G. (2002): Der mathematische Anfangsunterricht. Weinheim: Beltz.

Künzli, R. (2008): Schule als Ort des Wissens und seiner Bewertung. In: Zeitschrift für Erziehungswissenschaft 4, S. 405-414.

Lange, H. (1967): Schulbau und Schulverfassung der frühen Neuzeit. Zur Entstehung und Problematik des modernen Schulwesens. Weinheim: Beltz.

Latour, B. (2001): Eine Soziologie ohne Objekt? Anmerkungen zur Interobjektivität. In: Berliner Journal für Soziologie 11, S. 237-252.

MacKenzie, D. A./Wajcman, J. (Hrsg.) (1999): The Social Shaping of Technology. Buckingham: Open University Press.

Maier, M. S. (2014): Die Nähe/Distanz-Thematik in Lehrerkonferenzen. Eine organisationstheoretische Perspektive auf die Bearbeitung pädagogischer Probleme. In: Schulpädagogik heute 5, S. 1-16.

Marcus, G. E. (1998): Ethnography in/of the World System. The Emergence of Multi-Sited Ethnography. In: Ethnography Through Thick and Thin. Princeton, NJ: Princeton University Press, S. 79-104.

Mehan, H. (1979): Learning Lessons: Social Organization in the Classroom. Cambridge, MA: Harvard University Press.

Nadai, E. / Maeder, C. (2008): Negotiations at all Points? Interaction and Organization. In: Forum Qualitative Sozialforschung / Forum: Qualitative Social Research 9, abrufbar unter: http:/ / www.qualitative-research.net/index.php/fqs/article/view/337, letzter Zugriff am 13.6.2014.

Neyland, D. (2007): Organizational Ethnography. London: Sage.

Nohl, A.-M. (2011): Pädagogik der Dinge. Bad Heilbrunn: Klinkhardt.

Petrat, G. (1979): Schulunterricht. Seine Sozialgeschichte in Deutschland 1750-1850. München: Ehrenwirth.

Pille, T. (2013): Das Referendariat. Eine ethnographische Studie zu den Praktiken der Lehrerbildung. Bielefeld: Transcript.

Rammert, W./Schlese, M./Wagner, G./Wehner, J./Weingarten, R. (1998): Wissensmaschinen. Soziale Konstruktion eines technischen Mediums. Das Beispiel Expertensysteme. Frankfurt am Main: Campus.

Röhl, T. (2013): Dinge des Wissens. Schulunterricht als sozio-materielle Praxis. Stuttgart: Lucius \& Lucius.

Rosen, M. (1991): Coming to Terms with the Field: Understanding and Doing Organizational Ethnography. In: Journal of Management Studies 28, S. 1-24.

Schatzki, T.R. (2005): Peripheral Vision. The Sites of Organization. In: Organization Studies 26, S. 465-484.

Schein, E.H. (1984): Coming to a New Awareness of Organizational Culture. In: Sloan Management Review 25, S. 3-16.

Schiffler, H./Winkeler, R. (1994): Tausend Jahre Schule. Eine Kulturgeschichte des Lernens in Bildern. 4. Aufl. Stuttgart: Belser.

Schlieszeit, J. (2010): Whiteboard-Konzepte im Überblick. In: Computer+Unterricht 20, S. 3337.

Schmidt, R. (1967): Die geschichtliche Entwicklung des Volksschulbaues in Deutschland (1). In: Paedagogica Historica 7, S. 519-550.

Schmidt, R, / Volbers, J. (2011): Öffentlichkeit als methodologisches Prinzip. Zur Tragweite einer praxistheoretischen Grundannahme. In: Zeitschrift für Soziologie 40, S. 24-41.

Sørensen, E. (2009): The Materiality of Learning. Technology and Knowledge in Educational Practice. Cambridge: Cambridge University Press. 
Strauss, A.L. (1978): Negotiations. Varieties, Contexts, Processes, and Social Order. San Francisco: Jossey-Bass.

Vanderstraeten, R. (2001): The School Class as an Interaction Order. In: British Journal of Sociology of Education 22, S. 267-277.

Wiesemann, J./Lange, J. (2015) „Education in a Box“. Die Herstellung schulischer Artefakte in der Lehr-Lernmittelindustrie. In: Zeitschrift für interpretative Schul- und Unterrichtsforschung 4 (im Erscheinen).

Wernet, A. (2003): Pädagogische Permissivität. Schulische Sozialisation und pädagogisches Handeln jenseits der Professionalisierungsfrage. Opladen: Leske + Budrich.

Whyte, W. F. (1948): Human Relations in the Restaurant Industry. New York: McGraw-Hill.

Ybema, S. / Yanow, D. / Wels, H. / Kamsteeg, F. (Hrsg.) (2009): Organizational Ethnography: Studying the Complexity of Everyday Life. Thousand Oaks, CA: Sage. 